\title{
La objetualidad entre los videojuegos y el arte. Controles alternativos e instalaciones
}

Monica Jacobo ${ }^{(1)}$

Resumen: En festivales y exhibiciones de la industria de los videojuegos se presentan propuestas que incorporan elementos objetuales, aunque los juegos cada vez más frecuentemente se descargan de plataformas dedicadas a ese fin. Esas obras exploran la interfaz y en algunos casos se extienden por el espacio exhibitivo como instalaciones. Este trabajo explora las potencialidades de la objetualidad y su inclusión en eventos relacionados con los videojuegos, considerando los posibles recorridos y préstamos de obras, juegos y artistas entre los mundos del arte con tecnología y los de los videojuegos.

Las piezas que consideraremos para el desarrollo de esta propuesta son Line Wobbler, Buscador estelar de sueños y KIDS, producciones con distintas materialidades que se expusieron en Buenos Aires en la muestra Game On! 2019, espacio que tiene como particularidad reunir trabajos que se relacionan con la jugabilidad desde enfoques diversos.

Palabras clave: controles alternativos - interfaz - videojuegos - instalaciones - materialidad - objetualidad - festivales - arte interactivo - arte con tecnología - arte digital

[Resúmenes en inglés y portugués en la página 74-75]

(1) Dra. en Artes por la Universidad Nacional de Córdoba. Es investigadora y artista visual en el campo del arte y su vínculo con las tecnologías. Su interés se centra actualmente en los videojuegos como objetos culturales. Dirige un equipo de investigación sobre experiencias digitales: subjetividades, arte y cultura contemporánea en la Facultad de Artes. U.N.C.

\section{Introducción}

Los videojuegos son productos digitales que se pueden copiar, bajar, jugar y reproducir en distintos dispositivos. A partir del último cambio de siglo, la venta de copias materiales fue poco a poco sustituida por la distribución online, sin embargo, cada vez con más frecuencia en festivales y exhibiciones de la industria de los videojuegos se presentan propuestas que incorporan elementos objetuales. Estas son principalmente experimentaciones con la interfaz 
mediante la exploración de controles alternativos, pero también hay búsquedas que exploran la materialidad de las instalaciones, un lenguaje proveniente de los mundos del arte.

La categoría controles alternativos comenzó desde hace menos de una década a ser cada vez más frecuente en festivales y exhibiciones sobre videojuegos. La Game Developers Conference ${ }^{1}$, que cuenta con una amplia trayectoria en la industria del entretenimiento y sostiene en su misión institucional el "dar forma al futuro de la industria", incluye desde 2014 la exhibición Alt.Ctrl.GDC en la que se convoca a desarrolladores a presentar trabajos con dispositivos diferentes de las tradicionales interfaces que hacen posible la experiencia de jugar. El lema que utiliza esta sección en particular es cambiemos el modo en que jugamos ${ }^{3}$, objetivo en función del cual se invita a enviar a la convocatoria videojuegos con controladores "peculiares y excéntricos". Pantalla, mouse, teclado y joystick pueden ser reemplazados por propuestas que cubren un amplio abanico de investigaciones como la utilización de objetos cotidianos para la interacción o complejas y a veces curiosas interfaces construidas para ese fin. Los eventos que incluyen esta categoría en su line up, o que la presentan implícita bajo nodos curatoriales (Oulton, 2019) que requieren experimentaciones con la objetualidad, tienen lugar en numerosos espacios en el contexto internacional. El museo ZKM, institución que desde hace treinta años se dedica a las artes electrónicas y cuenta con exhibiciones efectuadas en torno a los videojuegos como eje, puso a consideración del público desde 2018 Gameplay. The Next Level ${ }^{4}$, que se desarrollará hasta el 2021 continuando a Gameplay, llevada a cabo algunos años antes. En la propuesta más reciente se incluyó un nodo de curación denominado creative \& aesthetic games que tenía por objetivo presentar ideas inusuales que expandieran las posibilidades de los juegos mediante la utilización de interfaces innovadoras o conectando el espacio virtual y el físico. A MAZE , festival que tiene lugar en Berlín desde el 2012, incluye investigaciones que amplían las posibilidades lúdicas mediante interfaces físicas y aproximaciones diferentes a las tradicionales en premiaciones con categorías que se fueron modificando con el correr de las ediciones. Las convocatorias al Explorer Award, Audience Award, y WTF?! Award buscaban, mediante la participación de los desarrolladores, "ir más allá de los límites de las formas contemporáneas de jugar o desarrollar juegos", incluyendo en el festival instalaciones interactivas, experimentos, performances robóticas y controles alternativos.

La muestra Videogames: Design/Play/Disrupt ${ }^{6}$, llevada a cabo entre el 2018 y 2019 en el museo londinense Victoria and Albert, institución enfocada en el diseño y sus diferentes expresiones, buscaba dar a conocer diversas aproximaciones sobre los videojuegos incorporando en su título la disrupción como una de sus metas.

Game On! El arte en juego, desarrollado en el contexto argentino, incluyó desde sus inicios en el 2009 las experimentaciones en la producción de videojuegos, así como interfaces físicas y creaciones de artistas tecnológicos.

Otros espacios como las game jams, entre las que se destaca la Alt Ctrl Game Jam ${ }^{7}$ en el contexto internacional, hackerlabs y diversas formas que adquiere la movida maker (Robles Miguélez, 2017), dan lugar a la participación con experimentaciones en torno a las interfaces de juegos en etapas tempranas de su realización. Estas son frecuentemente presentadas en estados anteriores al prototipo y testeadas de manera intensiva durante las sesiones que se montan en esos espacios de socialización y aprendizaje 
Los controles son una parte central de la interfaz, al ser esta el aspecto percibido por el jugador, su forma y comportamiento es lo que define la poética del videojuego, mientras que la programación permanece oculta. En la construcción de la interfaz para juegos, del mismo modo que para obras de arte interactivas, intervienen mandatos provenientes del diseño debido a su fin comunicacional, y se advierte la adhesión a búsquedas científicotecnológicas devenidas del propio uso de las tecnologías. La interfaz como elemento intermediario que hace posible la experiencia del usuario requiere seguir ciertos principios para poder cumplir su función. De acuerdo con Preece, J., Sharp, H. y Rogers, Y. (2002:21), estos son feedback, restricciones, mapping, consistencia y accesibilidad en su diálogo con el jugador. El primero consiste en generar información en respuesta a una acción, permitiendo al usuario continuar con una determinada actividad, el feedback puede ser auditivo, táctil, visual o combinaciones de estos. El segundo se refiere a establecer modos de restringir la interacción del usuario en un momento determinado, reduciendo las posibilidades de cometer errores. Se pueden clasificar en restricciones físicas, lógicas y culturales. El tercero se orienta a la relación entre los controles y sus efectos en la pantalla o ambiente interactivo las más usadas son las flechas de dirección: arriba, abajo, derecha e izquierda. El cuarto consiste en diseñar interfaces en las que mediante la ejecución de operaciones similares se obtengan resultados parecidos; la búsqueda se puede orientar a una consistencia externa manteniendo la coherencia con el modo en que funciona el mundo real o interna sosteniendo una lógica dentro del propio sistema o con otros similares. El quinto consiste en subrayar los atributos de un objeto que permiten al usuario conocer el modo de utilizarlo, brindando pistas perceptuales con el objetivo de facilitar la interacción. Estos principios de diseño, indispensables para un producto con fines comerciales, pueden ser tenidos en cuenta para realizar controles alternativos para videojuegos o por el contrario ser explícitamente contravenidos en función de la experimentación. La interfaz humanomáquina suele responder a paradigmas de interacción que los científicos de la informática tienen como metas de investigación; entre los observados por Preece, y otros (2002), resulta de interés el referido a bits tangibles o integración virtual y física, que consisten en dispositivos que tienden a combinar información digital con objetos físicos contribuyendo a la invisibilidad y la ubicuidad de la computación, dos paradigmas que complementan al primero. La finalidad última es aproximar los dos mundos, haciendo posible la manipulación de lo material convirtiendo los espacios que nos rodean en interfaces. La exploración de la interfaz como espacio de integración entre personas y computadoras se actualiza de manera permanente en tanto se crean productos para el consumo, ya sea este económico o simbólico. En los mundos de los videojuegos la materialidad se hace evidente dentro de la categoría controles alternativos, en tanto que otras formas objetuales se vinculan con las producciones que forman parte de la historia de los videojuegos como consolas o cabinas que se utilizaban en los arcades (Sellers, 2001). 


\section{Custom hardware: Line Wobbler}

Un trabajo contemporáneo que reúne varias de las posibilidades de sustitución de los dispositivos de la interfaz tradicional por controles alternativos es Line Wobbler (2015), de Robin Baumgarten, desarrollador independiente y artista de origen alemán que reside en Londres. El hardware como el software del juego fueron realizados íntegramente por el desarrollador, y consisten en una línea de leds que reemplaza la pantalla en la interacción con el usuario. En esta se condensa la abstracción de un juego dungeon crawler en la que el avatar es un punto verde, los enemigos puntos rojos, en tanto que el naranja y el blanco representan lava así como más peligros a sortear. El dispositivo de interacción que maneja el jugador es un tope de puerta flexible: mediante su accionar se avanza en el juego logrando que el punto verde se transforme en amarillo y pueda luchar contra los enemigos. La materialidad de la obra consiste en una tira de leds, lo que permite que la apariencia física del juego pueda adaptarse a distintos espacios de exhibición de acuerdo con la arquitectura en la que se instala, extendiéndose por la distribución de paredes o techos. La versión que pudo jugarse en Buenos Aires dentro de Game On 2019 se encontraba situada en el nodo game hacks y tomó la forma de un arco fijado a una estructura ortogonal de carácter escultórico. Mediante el uso de recursos mínimos, la propuesta cuestiona la búsqueda de realismo tridimensional en la industria de los videojuegos, en tanto que la supresión de la pantalla y su reemplazo por una sola línea de leds lo vuelve una pieza particular en la experimentación con la interfaz. La pantalla y su sustituto más utilizado, la proyección, son elementos constantes y escasamente reemplazados dentro de las propuestas experimentales en torno a los dispositivos de retroalimentación de los videojuegos. En cambio, en el terreno de las instalaciones interactivas que se despliegan en los mundos del arte con tecnología, es más frecuente que objetos creados para la interacción provean el feedback necesario para el interactor sin que medie la incorporación de pantallas o proyecciones.

La creaciones con controles alternativos que no siguen las convenciones clásicas (Becker, 2008) de los videojuegos, con las que los desarrolladores están acostumbrados a trabajar y el público a jugar, permiten a esas producciones innovadoras estar en una posición liminal que hace posible su exhibición en eventos vinculados a los videojuegos como en otros relacionados con los mundos del arte.

Baumgarten, al referirse a su obra en el contexto de Los dos lados de la pantalla, exposición que comenzó en Septiembre de 2019 en Fundación Telefónica de Madrid, la describe como una instalación: "Como desarrollador experimental de hardware de videojuegos, los museos y los actos públicos son una de las principales plataformas para exponer mis instalaciones. Estos juegos no se suelen poder comprar ni jugar en casa, sino solo en entornos como los museos" (Baumgarten, 2019). La instalación, un lenguaje que proviene de los mundos del arte que se afianzó dentro del arte conceptual y el minimalismo en particular, de ser una práctica marginal se transformó en epicentro de las prácticas institucionales hacia el cambio de siglo (Bishop, 2005), se despliega también dentro de los mundos de las artes con tecnologías. En el caso de Line Wobbler, el lenguaje de la instalación se desarrolla a partir de la posibilidad de ocupación espacial que determina su materialidad mediante la incorporación de tiras de leds que pueden sumarse y crear a la vez el espacio del juego y el de la instalación donde se desplaza el avatar e interactúa el espectador respectivamente. 
El carácter objetual de los juegos instalados impide copiarlos en un dispositivo, sin embargo, Line Wobbler tiene la particularidad de que el código fue divulgado por su autor en sitios donde se socializan tutoriales para realizar dispositivos que implican manipulación técnica de variado alcance. Al compartir el proceso de producción en las redes se extiende la dinámica de los hackerspaces, donde, "se presentan modos emergentes de conocimiento que actualizan la propuesta de democratización de la enseñanza a partir de una vivencia horizontal y compartida de comunicación (...) y se pasa al espacio compartido del taller; se sustituye la figura del maestro por la del colega con quien se aprende y a quien al mismo tiempo se enseña" (Rennó y Mattos, 2013, p. 1376)

En los mundos del arte se encuentra el debate siempre vigente en torno a qué parte de la obra debe realizar el artista. De acuerdo con Becker (2008), por un lado, la idea de que este debe tener ciertas habilidades técnicas para ser considerado como tal y, por otro, la creencia de que el productor simbólico solo debe aportar la idea y puede encargar lo demás a técnicos especialistas. El estatus de una actividad como central y artística puede variar según las circunstancias y pasar a considerarse de apoyo, o puede suceder, en sentido inverso, que un trabajo estimado de soporte se transforme en la actividad central. En el caso de Line Wobbler encontramos que si bien los aspectos técnicos se comparten dentro del mundo maker, el concepto de instalación quita el énfasis en la habilidad técnica del productor simbólico, subrayando la idea y es esta cualidad la que colabora en el ingreso hacia los mundos del arte.

Este trabajo efectuó un extenso recorrido desde el inicio en presentaciones y testeos en game jams, hackerlabs y publicaciones en redes, pasando por la participación en numerosos concursos y festivales donde obtuvo varios premios hasta exhibir y rentar su trabajo para eventos y museos. Baumgarten realizó en el 2017 otra obra titulada Wobble Garden que se puede considerar una secuela, en la que se utiliza nuevamente topes de puerta pero esta vez en gran cantidad, dando lugar a otra propuesta modular con posibilidades de adaptarse al ambiente expositivo. Esta última obra forma parte de la colección del museo ZKM en Karlsruhe.

\section{Buscador estelar de sueños, una instalación lumínica interactiva}

En el contexto de Game On! 2019, se exhibió Buscador estelar de sueños junto a Beyond the Garden, de la diseñadora argentina Laura Palavecino. Ambas obras se encontraban en el nodo Poéticas, aunque la primera no figuraba en el catálogo de la exposición. Se ubicaron una al lado de la otra y se podían observar apenas se ingresaba al espacio exhibitivo del festival debido al tamaño de las proyecciones de cada una, que se destacaba en la distribución. Buscador estelar de sueños se presentó por primera vez en 2016 como proyecto para la Bienal Kosice, evento que tuvo lugar en cuatro ocasiones, y se enfocaba en la relación entre arte y tecnología en derredor de la obra y poética de Gyula Kosice, referente de la agrupación Madí, uno de los movimientos artísticos no figurativos de la primera mitad del siglo XX en Argentina (García, 2011; Pacheco, 2003). En esa convocatoria, Buscador estelar de sueños se presentó como una instalación lumínica interactiva y obtuvo una mención del jurado. La obra desarrolla una poética personal en relación a la observación de las constelaciones, 
considerando que se constituyeron en la antigüedad como objetos guías para la ubicación geográfica, generadoras de momentos de contemplación de la naturaleza celeste y vínculo con mitologías ancestrales. Según palabras de la autora, las constelaciones utilizadas en la obra "no son las convencionales reconocidas por la Unión Astronómica Internacional, sino que son fruto del imaginario poético personal, que oficia como guía en una trayectoria artística propia" (Palavecino, 2019, p. 84). Los dibujos que se forman mediante líneas que unen las estrellas son animales de aspecto inocente vinculados a cuentos, mitos y una conocida fábula recreada en formato de videojuegos por la artista. Esas imágenes aparecen proyectadas frente al objeto que se utiliza como interfaz para la interacción con la obra que consiste en una maqueta de acrílico con la representación en tres dimensiones de una ciudad. En el centro de este se encuentra un dispositivo que se basa en el funcionamiento de un astrolabio. El aspecto del conjunto es brillante y luminoso ya que contiene luces leds que irradian por los bordes del acrílico donde la luz remarca los contornos. Mediante una manecilla en el astrolabio, cuya posición se puede modificar interactuando con las manos, la intervención del espectador/jugador produce cambios en las imágenes proyectadas permitiendo ver las distintas constelaciones propuestas por la autora. Palavecino tiene formación como diseñadora y en su trayectoria cuenta con la experiencia de haber sido seleccionada para participar en la Game Developers Conference dentro de la sección Alt.Ctrl.GDC, con un trabajo llamado Orpheus Quest, realizado en equipo junto a Raúl Palavecino y Aníbal Hormeche.

Buscador estelar de sueños se mostró en el contexto de los mundos del arte con tecnología en museos y exposiciones, así como en Game On!, cuya temática central son los videojuegos. No se puede bajar de internet ni es posible jugar con él en la intimidad de una habitación por las características materiales de su interfaz. La interacción se realiza en espacios públicos en una jugada corta que permite explorar las variantes existentes dentro de una propuesta poética interactiva.

La historia del arte interactivo, del que podemos situar sus inicios en la década de 1960 con los Experimentos en Arte y Tecnología (EAT), propulsada por la sinergia entre el ingeniero Billy Kluver y el artista Robert Rauschemberg en Estados Unidos con el auspicio de los laboratorios Bell (Herrera, 1997; Shanken, 2009), por citar una de las instancias más conocidas, tiene un importante acervo de obras en las que se ha trabajado y experimentado con la interfaz como objeto de comunicación estético conceptual. En Buscador estelar de sueños la objetualidad y su cuidado diseño incrementan la visibilidad del trabajo y el deseo de su apropiación mediante la interacción.

En esta obra, así como en Line Wobbler, los objetos funcionan como interfaz y dispositivos de entrada de datos en el aspecto técnico, realizando el pasaje del jugador desde fuera a dentro del juego/obra; en términos de Murray (1999): son objetos umbrales que mantienen al sujeto adentro de la obra y a la vez son la puerta de salida, el cordel en el laberinto hacia el mundo real. Estas experiencias transicionales que suponen pasar a percibir la narración o experiencia como real son muy frágiles frente a los videojuegos, que casi en su totalidad implican un cierto grado de narratividad e interacción al estar desarrollados con diferentes opciones de trama que dependen de las elecciones tomadas por el interactor (if/then en lenguaje de programación): la pregunta es como entrar al mundo ficcional sin romperlo. Los desarrolladores encontraron una solución a esta búsqueda mediante el 
recurso de utilizar objetos físicos como umbrales u objetos encantados, que sirven para penetrar en el juego, sus mecánicas y jugar con él.

\section{KIDS, videojuego, instalación y corto animado}

El juego KIDS (2019) es el segundo trabajo en colaboración entre Michael Frei, cineasta y artista, y Mario von Rickenbach, desarrollador de videojuegos, ambos de origen suizo. El primero fue un trabajo interactivo realizado en el año 2015 a partir de un corto llamado Plug \& Play. KIDS, y tiene en común con aquel el uso del blanco y negro como parte de su estética y la particularidad de poder exhibirse en más de un formato, ya que puede presentarse en el lenguaje del videojuego, como corto de animación o instalación. La posibilidad de desarrollar varias versiones de un mismo objeto es propio de los productos devenidos de las tecnologías digitales. De acuerdo con Manovic: "Es una consecuencia de la codificación numérica de los medios y de la estructura modular de los objetos mediáticos" (2006 p. 82). El trabajo realizado con dibujo lineal y movimiento fluido presenta personajes que habitan las distintas versiones del juego como multitudes anónimas, de forma antropomorfa pero sin rostro, ni ninguna característica o elemento que los distinga como individuos, parecen todos iguales. En el videojuego, que se puede adquirir en las plataformas de distribución más populares, la escena principal muestra un gran óvalo negro y gran cantidad de personajes en derredor. Si el jugador arrastra un personaje, la multitud lo sigue; irremediablemente se caen todos hacia algún otro lado, es posible detener algunos de ellos por un rato, pero ante nuestro abandono también se dejan ir. El corto de animación amplía los escenarios con escenas donde las multitudes actúan en conjunto, poniendo en imágenes ideas surrealistas o aludiendo a comportamientos sociológicos. KIDS se puede leer como un artgame de acuerdo con Sharp (2015, p. 54): "Estos tienden a basarse en una perspectiva rétorica particular sobre las relaciones, la tiranía del tiempo, la complicidad y otras ideas abiertas como exploraciones de la condición humana”.

El trabajo en el lenguaje de la instalación fue presentada por primera vez en 2018 en el Museo de Artes Digitales (MuDA) de Zúrich, un espacio que si bien está dedicado a la relación entre tecnologías y arte, propone que las pantallas no sean el nodo de las exposiciones y que se apele a otras sensaciones para experimentar las obras. En la exhibición se reunía las tres versiones: mostraba el juego en tres pequeñas pantallas, el corto era proyectado en una pared mientras que en todo el espacio exhibitivo se ubicaban muñecos blancos de tela similares a los que habitan el juego, esparcidos y amontonados en asientos o sobre el suelo donde se encontraba un círculo negro, posibilitando jugar con ellos.

El objetivo del MuDA de extender la experiencia digital más allá de las pantallas son de particular interés en relación con la objetualidad en tanto habilita otros modos de construir la percepción de la obra de arte con esas características, y es de ayuda en la justificación del salir de las pantallas cotidianas para asistir a un evento museístico.

KIDS se exhibió en numerosos festivales adquiriendo formas diversas que combinan sus tres formatos de acuerdo al espacio disponible. Dentro de Game On! se exhibió en el nodo Contactos, incorporando algunos personajes de tela sobre una estructura metálica en for- 
ma de pirámide trunca que se completaba con una pequeña pantalla y una proyección. El festival como modo de exhibición se caracteriza desde sus comienzos por su vínculo con la plurisensorialidad en cuanto a la recepción de la obra de arte, así como relacionado con lo festivo y con la posibilidad de percibir mediante una experiencia inmersiva una situación artística. Entre otros tempranos antecedentes encontramos el Festival de Bayreuth inaugurado en 1876 por Wagner, con la ayuda financiera de su benefactor Luis II de Baviera, para el cual se construyó el Festspielhaus, un teatro capaz de albergar las obras wagnerianas de acuerdo a su concepción de obra de arte total (Fubini, 1988). En tanto formato de exhibición y socialización actual, podemos situar al festival, entre las instituciones y las prácticas experimentales, en el linde entre la industria del entretenimiento y los mundos de arte. En su tejido se despliegan producciones de artistas noveles como de consagrados, nacionales e internacionales, que si bien comparten el espacio físico y simbólico, obtienen generalmente diferentes ubicaciones en las grillas de programación, así como en el espacio tomado por el festival. Dentro de este espacio los controles peculiares permiten la activación de otros sentidos junto al de la vista invitando al disfrute y a la socialización.

\section{Algunas consideraciones}

Los controles alternativos se transformaron en la última década en una convención (Becker, 2008) dentro de los mundos de los videojuegos, particularmente en los festivales y en las instancias previas que suelen recorrerse en el circuito de profesionalización entre los que se encuentran las game jams. El territorio para el cual muchos de ellos se desarrollan no es el de la reproducción masiva y comercialización en la industria del entretenimiento, sino otros recorridos en exhibiciones, festivales y museos, espacios públicos donde las obras son experimentadas ante su imposibilidad de hacerlo en instancias privadas. La materialidad con la que frecuentemente cuentan los controles ayuda a aumentar su visibilidad dentro de exhibiciones, donde la mayor parte de los productos son downloadables y reproducibles. Junto a estos desarrollos también encontramos otros con una disposición similar en cuanto al uso de la materialidad, juegos que se extienden por el espacio de las muestras en el lenguaje de la instalación permitiendo al espectador penetrar el área del juego para acceder a los objetos allí instalados antes de ingresar a su sección digital. Los tres trabajos analizados guardan un fuerte vínculo con el mundo del arte con tecnología. La primera exposición de KIDS, así como la de Buscador estelar de sueños, tuvieron lugar en contextos dentro de este mundo, el Museo de Arte digital (MUDA) en Zurich y la Bienal Kosice. El trabajo de Baumgarten se exhibió en una exposición temática sobre videojuegos en el museo ZKM, institución que posteriormente adquirió otra obra suya. Las materialidades de estas propuestas con jugadas generalmente cortas permiten una experiencia diferente que extiende el tiempo del juego y busca convertirse en una partida larga. 


\section{Notas:}

1. La Game Developers Conference se realiza desde 1988 en San Francisco, California. 2. "The Game Developers Conference (GDC) brings game development community together to exchange ideas, solve problems and shape the future of the industry during five days of education, inspiration, and networking. The attendees list includes programmers, artists, producers, game designers, audio professionals and industry leaders."

https://www.gdconf.com/about

3. "Change the way we play"

4. Gameplay-the-next-level. https://zkm.de/en/exhibition/2018/09/zkmgameplay-the-nextlevel

5. A MAZE. https://amaze-berlin.de/

6. Videogames: Design/Play/Disrupt. https://www.vam.ac.uk/exhibitions/videogames

7. Alt Ctrl Game Jam. https://altctrlgamejam.com/

8. Los dos lados de la pantalla. https://espacio.fundaciontelefonica.com/evento/inauguracion_videojuegos_la_expo/

\section{Referencias bibliográficas}

Baumgarten, R. (2019). El desarrollo experimental de videojuegos. Recuperado de: https:// espacio.fundaciontelefonica.com/noticia/desarrollo-experimental-de-videojuegoscomo-romper-los-limites-por-robin-baumgarten/?ide $=64838$

Becker, H. (2008). Los mundos del arte: Sociología del trabajo artístico. Buenos Aires: Universidad Nacional de Quilmes.

Bishop, C. (2011). Installation Art. Londres: TATE publishing.

Fubini, E. (1988). La estética musical desde la Antigüedad hasta el siglo XX. Madrid: Alianza.

García, M. A. (2011). El arte abstracto. Intercambios culturales entre argentina y Brasil. Buenos Aires: Siglo Veintiuno Editores.

Herrera, M. J. (1997). En medio de los medios. La experimentación con los medios masivos de comunicación en la Argentina de la década del sesenta. Arte argentino del siglo XX, Premio Telefónica de Argentina a la Investigación en Historia de las Artes Plásticas (pp. 69-114). Buenos Aires: Fundación Telefónica.

Manovich, L. (2006). El lenguaje de los nuevos medios de comunicación: La imagen en la era digital. Buenos Aires: Paidós.

Murray, J. (1999). Hamlet on the Holodeck. Cambridge: The MIT Press.

Oulton, L. (2019). Videojuegos en el museo. Nuevos desafíos curatoriales. Cuadernos 98, 93-106. Buenos Aires: Universidad de Palermo.

Pacheco, M. (2003). Arte Abstracto Argentino. Buenos Aires: Fundación Proa.

Palavecino, L. (2019). Videojuegos, arte, naturaleza y maravilla. Un análisis transdisciplinar sobre las posibilidades poéticas de los nuevos medios. Cuadernos 98, 75-92. Buenos Aires: Universidad de Palermo. 
Preece, J., Sharp, H. y Rogers, Y. (2002). Interaction Design. Beyond human computer interaction. New York: John Wiley.

Rennó R.y Mattos C. (2013). Activismo en Brasil: los hackerspaces como espacios de resistencia y enseñanza libre. Acta final del II Congreso Internacional Educación mediática \& competencia digital: Ludoliteracy, creación colectiva y aprendizajes (pp.1372-1383). Barcelona: Obra social La Caixa. Recuperado de: https://www.uoc.edu/portal/es/symposia/congreso_ludoliteracy2013/conclusiones_actas/ACTAS_DEFINITIVAS_CONGRESO_EDUMED_2013.pdf

Robles Miguélez N. (2017). Makers, fabricantes de ideas. Muriel D. y San Salvador del Valle R.(eds.) Tecnología digital y nuevas formas de ocio (pp.73-84). Bilbao: Universidad de Deusto.

Sellers J. (2001). Arcade Fever. The Fan's Guide To The Golden Age Of Video Games. Philadelphia: Running Press.

Shanken, E. (2009). Art and electronic media. New York: Phaidon.

Sharp, J. (2015). Works of Game: On the Aesthetics of Games and Art. Cambridge: The MIT Press.

\begin{abstract}
Festivals and exhibitions in the video game industry feature proposals that incorporate object elements, although games are increasingly downloaded from dedicated platforms. These works explore the interface and in some cases extend into the exhibition space as installations. This work explores the potential of objectivity and its inclusion in videogame-related events, considering the possible routes and loans of works, games and artists between the worlds of art with technology and those of videogames.

The pieces that we will consider for the development of this proposal are Line Wobbler, Star Searcher of Dreams and KIDS, productions with different materialities that were exhibited in Buenos Aires in the exhibition Game On! 2019, a space that has the particularity of gathering works that are related to gameplay from different approaches.
\end{abstract}

Keywords: alternative controls - interface - video games - installations - materiality objecthood - festivals - interactive art - art with technology - digital art

Resumo: Festivais e exposições na indústria dos videojogos apresentam propostas que incorporam elementos objecto, embora os jogos sejam cada vez mais descarregados a partir de plataformas dedicadas. Estes trabalhos exploram a interface e em alguns casos estendemse ao espaço expositivo como instalações. Este trabalho explora o potencial da objetividade e sua inclusão em eventos relacionados a videogames, considerando as possíveis rotas e empréstimos de obras, jogos e artistas entre o mundo da arte com tecnologia e o dos videogames. 
As peças que iremos considerar para o desenvolvimento desta proposta são Line Wobbler, Star Searcher of Dreams e KIDS, produções com diferentes materialidades que foram expostas em Buenos Aires na exposição Game On! 2019, um espaço que tem a particularidade de reunir trabalhos que estão relacionados com a jogabilidade a partir de diferentes abordagens.

Palavras chave: controlos alternativos - interface - jogos de vídeo - instalações materialidade - objectividade - festivais - arte interactiva - arte com tecnologia - arte digital

[Las traducciones de los abstracts fueron supervisadas por el autor de cada artículo] 
